\title{
Large-Scale Hypoconnectivity Between Resting-State Functional Networks in Unmedicated Adolescent Major Depressive Disorder
}

\author{
Matthew D Sacchet, ${ }^{* 1,2}$, Tiffany C Ho ${ }^{2}$, Colm G Connolly ${ }^{2}$, Olga Tymofiyeva ${ }^{3}$, Kaja Z Lewinn ${ }^{2}$, \\ Laura KM Han ${ }^{2,4}$, Eva H Blom ${ }^{2,5}$, Susan F Tapert ${ }^{6}$, Jeffrey E Max ${ }^{6}$, Guido KW Frank ${ }^{7,8}$, Martin P Paulus ${ }^{6,9}$, \\ Alan N Simmons ${ }^{6,10}$, lan H Gotlib' and Tony $\mathbf{T ~ Y a n g}^{2}$ \\ 'Neurosciences Program and Department of Psychology, Stanford University, Stanford, CA, USA; '2Department of Psychiatry, Division of Child and \\ Adolescent Psychiatry, University of California San Francisco, San Francisco, CA, USA; ${ }^{3}$ Department of Radiology and Biomedical Imaging, University \\ of California San Francisco, San Francisco, CA, USA; ${ }^{4}$ Institute of Interdisciplinary Studies, Amsterdam Brain and Cognition, University of Amsterdam, \\ Amsterdam, The Netherlands; ${ }^{5}$ Department of Clinical Neuroscience, Karolinska Institutet, Solna, Sweden; ${ }^{6}$ Department of Psychiatry, University of \\ California San Diego, La Jolla, CA, USA; ${ }^{7}$ Department of Psychiatry, University of Colorado, Anschutz Medical Campus, Aurora, CO, USA; \\ ${ }^{8}$ Neuroscience Program, University of Colorado, Anschutz Medical Campus, Aurora, CO, USA; ${ }^{9}$ Laureate Institute for Brain Research, Tulsa, OK, \\ USA; ${ }^{10}$ Center of Excellence for Stress and Mental Health, Veterans Affairs San Diego Health Care System, San Diego, CA, USA
}

\begin{abstract}
Major depressive disorder (MDD) often emerges during adolescence, a critical period of brain development. Recent resting-state fMRI studies of adults suggest that MDD is associated with abnormalities within and between resting-state networks (RSNs). Here we tested whether adolescent MDD is characterized by abnormalities in interactions among RSNs. Participants were 55 unmedicated adolescents diagnosed with MDD and 56 matched healthy controls. Functional connectivity was mapped using resting-state fMRI. We used the network-based statistic (NBS) to compare large-scale connectivity between groups and also compared the groups on graph metrics. We further assessed whether group differences identified using nodes defined from functionally defined RSNs were also evident when using anatomically defined nodes. In addition, we examined relations between network abnormalities and depression severity and duration. Finally, we compared intranetwork connectivity between groups and assessed the replication of previously reported MDD-related abnormalities in connectivity. The NBS indicated that, compared with controls, depressed adolescents exhibited reduced connectivity ( $p<0.024$, corrected) between a specific set of RSNs, including components of the attention, central executive, salience, and default mode networks. The NBS did not identify group differences in network connectivity when using anatomically defined nodes. Longer duration of depression was significantly correlated with reduced connectivity in this set of network interactions ( $p=0.020$, corrected), specifically with reduced connectivity between components of the dorsal attention network. The dorsal attention network was also characterized by reduced intranetwork connectivity in the MDD group. Finally, we replicated previously reported abnormal connectivity in individuals with MDD. In summary, adolescents with MDD show hypoconnectivity between large-scale brain networks compared with healthy controls. Given that connectivity among these networks typically increases during adolescent neurodevelopment, these results suggest that adolescent depression is associated with abnormalities in neural systems that are still developing during this critical period.
\end{abstract}

Neuropsychopharmacology (2016) 4I, 295 I-2960; doi: I 0.1038/npp.20I6.76; published online I5 June 2016

\section{INTRODUCTION}

Major depressive disorder (MDD) is the leading cause of disability and morbidity in teenagers and young adults worldwide (Jamison et al, 2006), with an estimated lifetime prevalence of $24 \%$ by age 25 years (Lewinsohn et al, 1998).

\footnotetext{
*Correspondence: MD Sacchet, Neurosciences Program and Department of Psychology, Stanford University, Jordan Hall, Building OI-420, 450 Serra Mall, Stanford, CA 94305, USA, Tel: +I 650723 0804, E-mail: msacchet@stanford.edu

Received I5 October 2015; revised 14 May 2016; accepted 17 May 2016; accepted article preview online 26 May 2016
}

Compared with adult-onset MDD, adolescent-onset MDD is associated with an increased likelihood of recurrent depressive episodes, increased symptom severity, and higher risk of suicide throughout the lifespan (Birmaher and Brent, 2007; Birmaher et al, 2002; Jamison et al, 2006; Kessler and Walters, 1998). Moreover, adolescence is a period of marked neurodevelopment in functional brain networks (Fair et al, 2008, 2009; Supekar et al, 2009); recent evidence indicates that mood disorders such as MDD may alter the neurodevelopmental trajectories of these networks (Ho et al, 2015). Few studies, however, have investigated empirically the effects of MDD on functional brain networks in adolescence. 
Researchers have used resting-state functional connectivity methods to assess functional brain networks; these methods index the strength of correlation between pairs of brain regions during task-free conditions. Studies of resting-state functional connectivity have documented widespread abnormalities in depressed adolescents (for a review, see Kerestes et al, 2010) and adults (for a review, see Mulders et al, 2015). These abnormalities typically involve regions associated with intrinsic resting-state networks (RSNs): the frontoparietal central executive network (including dorsolateral prefrontal cortex), the cingulo-opercular salience network (including cingulate and insular cortex), and the default mode network (including medial prefrontal, posterior cingulate cortex, and the precuneus) (Menon, 2011). Several studies of adolescent MDD have assessed the functional connections of a single region, or 'seed'. Seed-based approaches are useful for assessing how a given seed region is functionally connected to the rest of the brain or to specific other brain regions but do not allow for the assessment of functional interactions among multiple brain regions or networks. The brain is composed of large-scale functional networks that integrate signals from multiple regions to facilitate effective information processing; network-based approaches allow the characterization of this large-scale integration (Rubinov and Bullmore, 2013).

'Brain connectomics' is the study of comprehensive maps of structural and functional connections in an organism's central nervous system (Rubinov and Bullmore, 2013). In this framework, whole brain connectivity is modeled as a network (or graph), with brain regions as nodes and functional or structural connections as edges. The resulting networks can be compared using summary network measures or at the level of a single connection or sets of connections. The latter type of comparison can be performed, for example, using the network-based statistic (NBS), which offers a powerful approach that characterizes differences in the interconnectedness between multiple regions across sets of networks (Zalesky et al, 2010). Relatedly, graph theoretical analysis permits the calculation of summary measures that quantify various characteristics of network topology and organization (Fornito et al, 2013). These metrics permit the detailed characterization of largescale network properties (see section 'Graph Metric Computation' in Supplementary Materials for descriptions and properties of several graph metrics). Network properties can thus be assessed in relation to the whole brain or specific regions and can be used to understand abnormalities that may be important in characterizing psychiatric and neurological dysfunction.

To date, only one preliminary study that included 12 depressed adolescents has used graph theory to examine connectivity in adolescent MDD (Jin et al, 2011), and no investigations have yet used the NBS or graph theory to study interactions among RSNs in depressed adolescents. Jin et al (2011) reported widespread MDD-related increases in the graph metric of nodal degree (i.e., the number of connections of a region) and MDD-related decreases in the graph metric of small-worldness, which indicates that the MDD group has some combination of less dense local clustering of connections and longer path lengths between distant pairs of nodes compared with the CTL group (Bassett and Bullmore, 2006). Importantly, these findings appear to contradict most prior graph theoretical resting-state studies in adult MDD, which have found no differences between MDD and healthy controls in small-worldness (Bohr et al, 2012; Meng et al, 2014; Ye et al, 2015; Zhang et al, 2011) or nodal degree (Bohr et al, 2012; Lord et al, 2012; Meng et al, 2014; Zhang et al, 2011). Moreover, even within studies of adult MDD that have used the NBS and graph theory, results are inconsistent, perhaps reflecting the heterogeneity of the patient samples with respect to such factors as age, age of depression onset, number of depressive episodes, comorbidities, and medication. Furthermore, almost all of the NBS and graph studies of MDD (adolescent and adult) to date, including the study by Jin et al (2011), used anatomically defined nodes. Such nodes may not have homogenous signal if they do not fall within a single functional region and therefore may be suboptimal for characterizing functional network properties (Gordon et al, 2014). Thus further research with larger samples and functionally defined nodes is needed to assess the replicability of these preliminary findings in depressed adolescents, to determine possible concordance in functional network abnormalities between adolescent and adult MDD, and to identify meaningful and potentially clinically significant neural abnormalities in individuals with MDD.

The primary goal of this study was to assess abnormalities among interactions between RSNs by applying the NBS and graph theory to resting-state data from a large cohort of adolescents with MDD compared with well-matched healthy controls (CTL). Based on the preliminary graph theory study of adolescent depression and prior network studies of adult MDD, we hypothesized that adolescents with MDD would be characterized by large-scale functional abnormalities in connectivity among RSNs theorized to be adversely affected by MDD, including the central executive, salience, default mode, and limbic networks (Kerestes et al, 2010; Mulders et al, 2015). Specifically, we hypothesized decreased connectivity between the central executive and other networks in MDD vs CTL and increased network connectivity between the limbic and other networks. Based on the preliminary findings by Jin et al (2011), we hypothesized reduced smallworldness and increased intranetwork nodal degree of the limbic network in MDD vs CTL. Because anatomically defined nodes have been used most commonly in graph theoretical studies of MDD (Gong and He, 2015), we also conducted analyses using nodes defined from anatomical parcellation to further contextualize group differences in network properties identified using nodes from functionally defined from RSNs. In addition, within the group of MDD adolescents, we examined whether network abnormalities were related to either the severity or duration of depression. To provide a more comprehensive analysis, each of the 17 nodes was broken down into smaller nonadjacent components and between-group differences in intranodal functional connectivity were assessed. Finally, to assess replication of previous findings from our group (Connolly et $a l, 2013$ ) and concordance with meta-analytic results from the adult MDD literature (Kaiser et al, 2015), we conducted a series of additional seed-based functional connectivity analyses. 
a
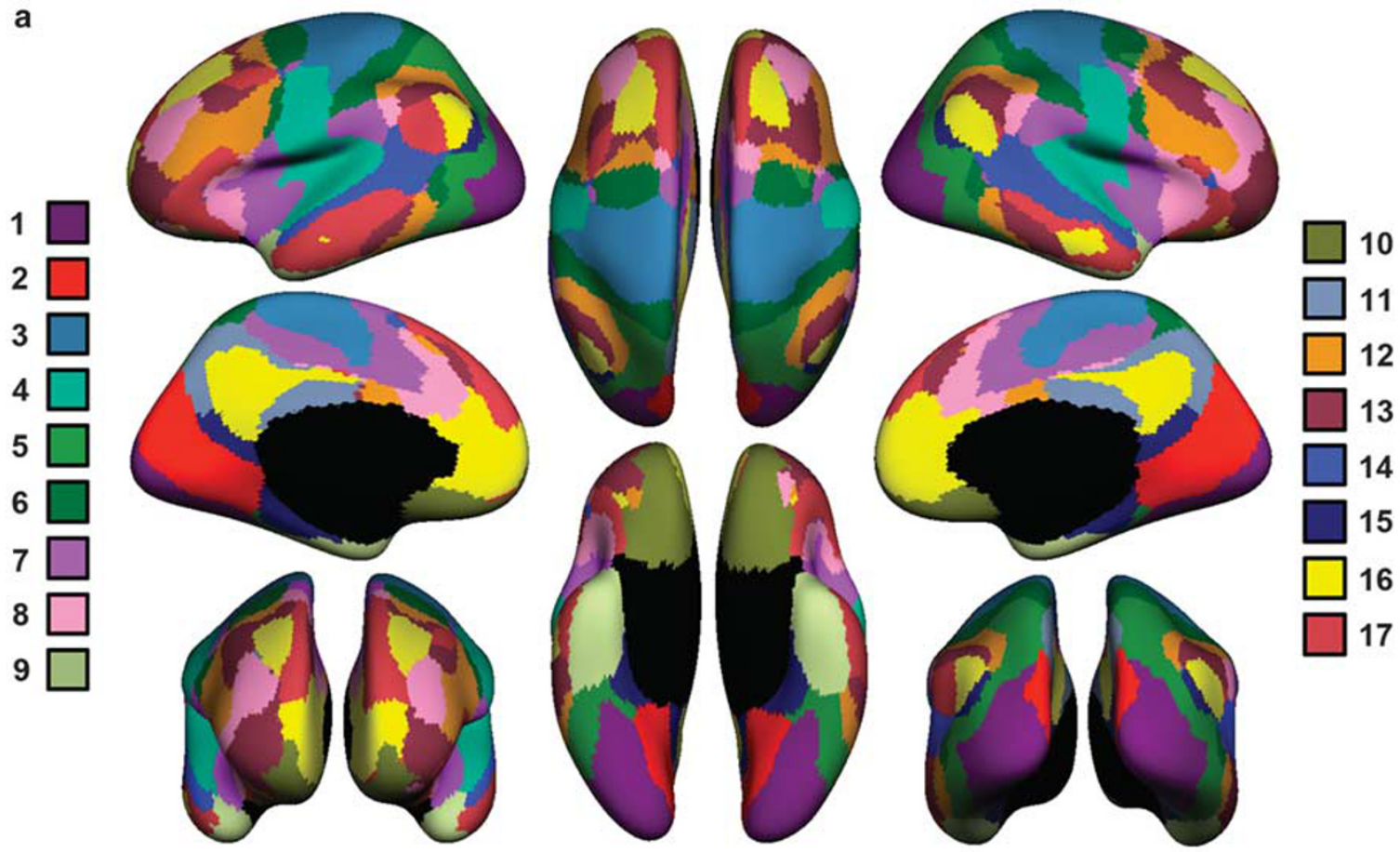

b

1. Data Acquisition 2. Preprocessing

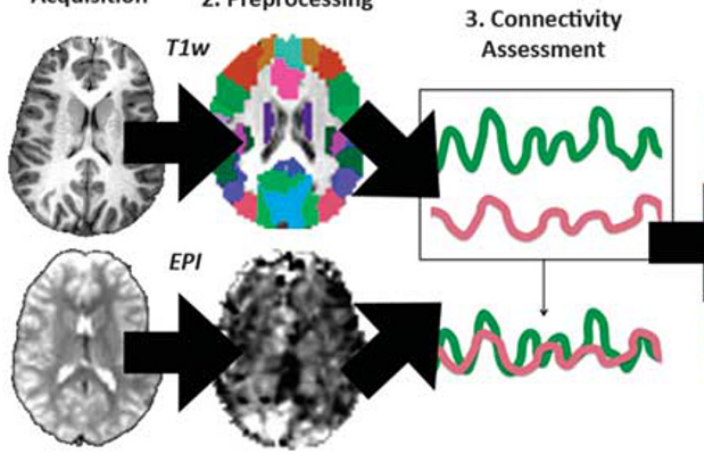

4. Correlation Matrix

5. Network Analysis
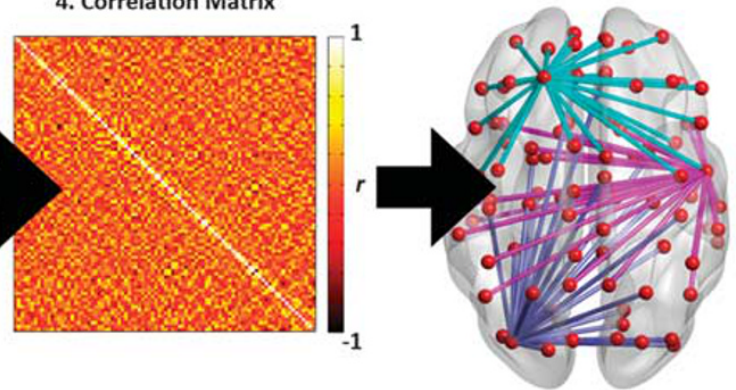

Figure I Resting-state networks (RSNs) and processing pipeline. (a) I7-network solution RSNs used as nodes in the network analyses by Yeo et al (20 I I). Multiple views of each hemisphere of cortical renderings of the 17-network solution RSNs by Yeo et al (20I I). Each color represents a different I7-network solution RSN. See Table I for RSN functional labels. (b) (I) TI-weighted (TIW) and echo planar fMRI images (EPIs) were acquired using an MRI system. (2) TIW and EPI images were preprocessed, including normalization to MNI space. (3) Time courses were extracted from each RSN (nodes), and functional connectivity was assessed for all pairs of RSNs (edges). (4) This resulted in a $17 \times 17$ correlation matrix for each participant. (5) These correlation matrices were used in subsequent network analyses using the network-based statistic (NBS) and graph theory.

\section{MATERIALS AND METHODS}

\section{Participants and Clinical Information}

Study protocol, recruitment procedures, clinical and diagnostic assessments, and inclusion/exclusion criteria have been previously described (Connolly et al, 2013; Ho et al, 2015) and are also detailed in 'Participants and Clinical Information' in Supplementary Materials. Data from 55 unmedicated MDD and 56 CTL adolescents were included in the current study. Participants were aged between 13 and 18 years, right handed, and physically healthy. All participants and their parent(s)/legal guardian(s) provided written informed assent and consent, respectively, in accordance with the Declaration of Helsinki. The Institutional Review Boards at the University of California San Diego, University of California San Francisco, Rady Children's Hospital in San Diego, and the County of San Diego approved this study.

\section{MRI Data Acquisition}

Data were collected using a 3T MRI system (MR750, GE Healthcare, Milwaukee, Wisconsin). T1-weighted (T1w) images were collected for anatomical registration and localization. Resting-state fMRI data were collected using echo planar imaging (EPI). See 'MRI Data Acquisition' in Supplementary Materials for details. 
Table I Yeo 17-Network Solution Functional Labels

\begin{tabular}{ll}
\hline Yeo I7-network solution value & Network labels \\
\hline 1 & Visual \\
2 & Visual \\
3 & Somatomotor \\
4 & Somatomotor \\
5 & Attention \\
6 & Attention \\
7 & Salience \\
8 & Salience \\
9 & Limbic \\
10 & Limbic \\
11 & Central executive \\
12 & Central executive \\
13 & Central executive \\
14 & Default mode \\
15 & Default mode \\
16 & Default mode \\
17 & Default mode \\
\hline
\end{tabular}

We derived labels for each of the 17 networks in the 17-network solution by Yeo et al $(201 \mathrm{I})$ by assessing the degree of overlap between the 17 networks with the 7-network solution, which is comprised of well-established resting-state networks (RSNs). See 'Functional Labels for the Yeo 17-Network Solution

Networks' and Supplementary Table S2 in the Supplementary Materials for more information.

\section{MRI Data Preprocessing}

Briefly, EPI data were despiked, slice-time and motion corrected, normalized, filtered, and smoothed. Effects from nuisance covariates were mitigated using multiple linear regression. Data associated with excessive movement were removed. Individuals with excessive movement were excluded from analysis. See 'MRI Data Preprocessing' in Supplementary Materials for details. We conducted a control analysis and found that between-group network differences were not attributable to motion-related group differences (see 'Control Analyses for Movement and Censored Volumes' in Supplementary Material).

\section{Network Node and Edge Definition}

Networks are defined as sets of nodes and their edges. To identify functional relations among RSNs, here RSNs represented nodes, and edges were represented by statistical interdependence in BOLD signals between RSNs. RSNs were defined based on the 17-network solution from Yeo et al (2011) projected to MNI152 space. See Figure 1a for RSN renderings and Table 1 for RSN labels, and section 'Functional Labels for the Yeo 17-Network Solution Networks' and Supplementary Table S2 in Supplementary Materials for information on functional label derivation. For edge definition, as is commonly performed, functional connectivity was computed for every pair of RSNs using partial correlation coefficients, while accounting for signal from all other RSNs. See Figure $1 b$ for a summary of the analysis pipeline.

\section{Calculation of the NBS}

We used the NBS to identify large-scale interactions among RSNs that differentiated the MDD from the CTL group (Zalesky et al, 2010). The NBS controls the family-wise error rate when conducting mass-univariate tests across the connections of a graph. Similar to cluster-extent methods in mass-univariate analyses of fMRI data, NBS identifies the extent of interconnected edges that are statistically associated with a given contrast. Because NBS assesses a whole-graph property (i.e., number of interconnected edges) instead of edges individually, it offers substantial increases in statistical power over methods that correct for multiple comparisons across tests at all edges individually. See 'Calculation of the Network-Based Statistic' in Supplementary Materials for more details.

\section{Global and Local Graph Metrics}

Global graph metrics summarize whole-graph properties. Local graph metrics characterize single nodes in a graph based on the network characteristics related to that node. Global and local graph metrics were computed from binarized correlation matrices using an adaptive thresholding procedure (see 'Network Binarization' in Supplementary Materials for details). We computed the global graph metrics of (1) clustering coefficient, (2) characteristic path length, (3) small-worldness, and (4) global efficiency, and the local graph metrics of (1) degree, (2) betweenness centrality, and (3) local efficiency (as in Korgaonkar et al, 2014; Zhang et al, 2011). See 'Graph Metric Computation' in Supplementary Materials for equations used and details regarding the network properties that these metrics assess. Nonparametric permutation-based statistical tests were used to assess group differences in each graph measure (Bullmore et al, 1999) (see 'Group Differences in Global and Local Graph Metrics' in Supplementary Material for details).

\section{Anatomically Defined Nodes}

To date, the anatomically defined AAL atlas (TzourioMazoyer et al, 2002) is the most commonly used node definition for the assessment of graph metrics in MDD (Gong and $\mathrm{He}, 2015$ ). Here, to conduct a more comprehensive analysis, we also assessed network differences between groups using nodes defined from the AAL atlas. We conducted these analyses using the same methods that were described for the analyses with nodes based on the Yeo 17-network solution (Yeo et al, 2011).

\section{Correlations Between Network Metrics and Clinical Measures and Age}

For all network metrics on which the MDD and CTL groups differed significantly, we computed correlations within the MDD group between that metric and the severity and duration of depression. Severity of depression was measured as total $T$-scores on the Reynolds Adolescent Depression Survey-Version 2 (RADS-2) (Reynolds and Mazza, 1998). Duration of depression was defined as the difference in each participant's current age and the integer age in years at which they reported that their first depressive episode occurred. 


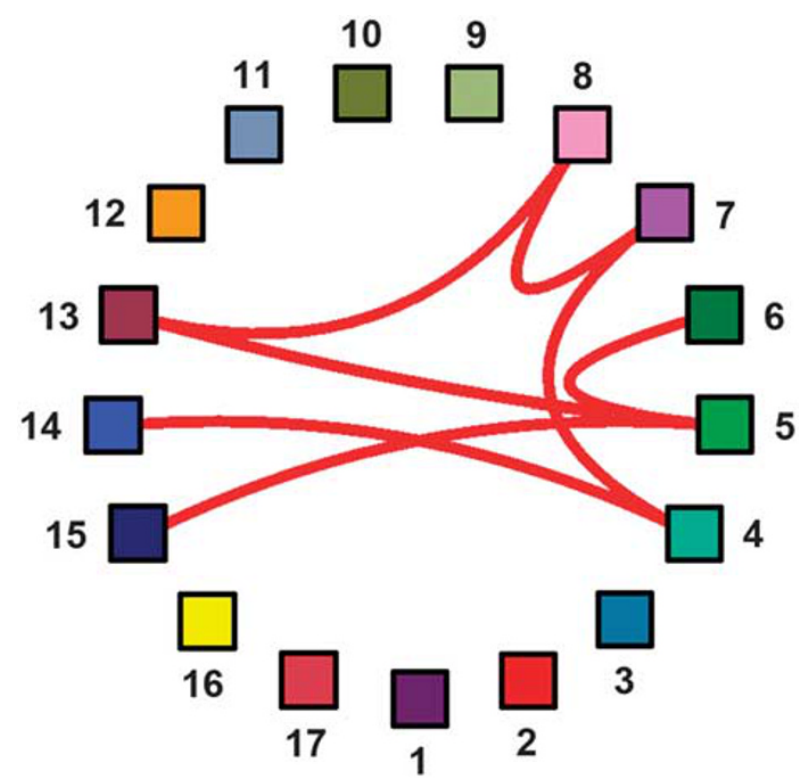

Figure 2 Results of the network-based statistic (NBS). Schemaball representation of reduced connectivity between resting-state networks (RSNs) in the MDD compared with the CTL group. Network colors and numbers (associated with a given node represented as a square) correspond with those presented in Figure I. Red lines indicate reduced connectivity in the MDD vs CTL group between the given networks identified using NBS. All results controlled for age, gender, and levels of intelligence. See Table 2 for network labels and Table 3 for details on results from the NBS.

Pearson correlation was used except when either variable was non-normally distributed as assessed by the Lilliefors test $(p<0.05)$, in which case Spearman's rank correlation was used. One participant was missing RADS-2 scores (resulting in $n=54$ ), and nine participants did not provide information for calculation of depression duration $(n=46)$. Three participants reported multiple values (i.e., a range, or uncertainty) for the age at which their first depressive episode occurred. For these cases, duration was computed as the average of these values. Both within and across groups, analyses were conducted to test whether network properties that were associated with MDD were related to age. All correlation analyses were conducted in MATLAB (The MathWorks, Natick, MA).

\section{Intranodal Connectivity}

Additional analyses were conducted to assess intranodal connectivity for all the 17 nodes. Spatially distinct components of each node were identified from the Yeo 17network solution using a connected components analysis (Yeo et al, 2011). Two-sample $t$-tests were used to assess significance of the difference between correlation coefficients that were $z$-transformed. Multiple linear regression was used before significance testing to mitigate effects related to age, gender, and Wechsler Abbreviated Scale of Intelligence (WASI) total scores. Intranodal analyses were conducted using MATLAB (The MathWorks).

\section{Correcting for Multiple Comparisons}

Bonferroni correction was used to account for false positive inflation from multiple statistical comparisons. We used the
Table 2 Results of the Network-Based Statistic (NBS)

\begin{tabular}{|c|c|c|c|}
\hline Network I & Network 2 & t-Value & $p$-Value \\
\hline Network 5 (Attention) & Network 6 (Attention) & 1.94 & 0.027 \\
\hline Network 4 (Somatomotor) & Network 7 (Salience) & 1.77 & 0.039 \\
\hline Network 7 (Salience) & Network 8 (Salience) & 2.45 & 0.008 \\
\hline Network 5 (Attention) & Network I3 (Central executive) & 2.26 & 0.013 \\
\hline $\begin{array}{l}\text { Network I } 3 \text { (Central } \\
\text { executive) }\end{array}$ & Network 8 (Salience) & 1.76 & 0.041 \\
\hline Network 4 (Somatomotor) & Network 14 (Default mode) & 2.51 & 0.007 \\
\hline Network 5 (Attention) & Network 15 (Default mode) & 2.09 & 0.019 \\
\hline
\end{tabular}

The identified network is described in terms of the direction of the group effect, the total number of nodes and edges, and the nodes that each edge connected. Statistics are presented from the NBS permutation test and from the independent-sample one-sided $t$-tests for each edge $(d f=109)$. The network was characterized by greater connectivity in the CTL than in the MDD group (permutation test, $p=0.0237$ ). This NBS-identified network included eight nodes and seven edges. See Figure 2 for visual representation of connectivity.

NBS to assess network abnormalities that were characterized by greater connectivity in the MDD group than in the CTL group and less connectivity in the MDD group than in the CTL group. To account for these multiple tests, $\alpha$ was set to $0.025(0.05 / 2)$. As in Korgaonkar et al (2014), Bonferroni correction was used for tests of global graph metrics $(\alpha=0.0125 ; 0.05 / 4)$ and again for each local graph metric across all regions $(\alpha=0.00294 ; 0.05 / 17)$ (Korgaonkar et al, 2014). For the correlational analyses, tests were corrected at the level of clinical measures $(\alpha=0.025 ; 0.05 / 2)$ and then again in follow-up analyses that were used to assess relations between clinical variables and specific connections between RSNs ( $\alpha=0.05 / n$, where $n=$ number of connections). Bonferroni correction was also used for the intranodal analyses such that all tests for each node were corrected. For example, node 2 (visual network) was composed of 6 discontinuous subcomponents and resulted therefore in 15 total tests, with a significance threshold of $p=0.003$ (i.e., 0.05/15).

\section{Replication of Connolly et al (2013) and Kaiser et al (2015)}

We conducted a series of seed-based analyses in order to assess replication of Connolly et al (2013) and Kaiser et al (2015). See Supplementary Methods for methodological details ('Replication of Connolly et al (2013)', 'Replication of Kaiser et al (2015)').

\section{RESULTS}

\section{Demographic and Clinical Characteristics}

The MDD and CTL groups did not differ significantly in age, gender, pubertal stage, or socioeconomic status (all $p s \geqslant 0.25$; Supplementary Table S1). As expected, the MDD group exhibited higher levels of depression and anxiety (all $p s<0.001$ ). The CTL group obtained higher scores on the WASI than did the MDD group $(p<0.001)$. For subsequent analyses, network measures were regressed on age, gender, and WASI scores, and the resulting residuals were used in group-level analyses (e.g., as in Bai et al, 2012; Zhang et al, 2011; see Supplementary Material 'Calculation of the Network-Based Statistic' and 'Group Differences in 
Table 3 Correlations of Network-Based Statistic (NBS) Results with Clinical Variables

\begin{tabular}{|c|c|c|c|c|}
\hline Network measure & & Clinical metric & $\rho$ & p-Value \\
\hline \multicolumn{5}{|l|}{ Mean connectivity of intemetwork } \\
\hline \multicolumn{2}{|l|}{ Mean connectivity of internetwork } & Depression duration & $-0.344^{\mathrm{a}}$ & $0.020^{b}$ \\
\hline \multicolumn{2}{|l|}{ Mean connectivity of internetwork } & RADS-2 total T-score & $0.120^{\mathrm{a}}$ & 0.387 \\
\hline \multicolumn{5}{|c|}{ Single edge connectivity } \\
\hline Network 5 (Attention) & Network 6 (Attention) & Depression duration & $-0.425^{a}$ & $0.003^{c}$ \\
\hline Network 4 (Somatomotor) & Network 7 (Salience) & Depression duration & 0.002 & 0.989 \\
\hline Network 7 (Salience) & Network 8 (Salience) & Depression duration & -0.269 & 0.070 \\
\hline Network 5 (Attention) & Network 13 (Central executive) & Depression duration & 0.168 & 0.264 \\
\hline Network 13 (Central executive) & Network 8 (Salience) & Depression duration & -0.048 & 0.749 \\
\hline
\end{tabular}

Global and Local Graph Metrics'). For the correlations computed between age and network properties, the network properties were regressed only on gender and WASI scores.

\section{Results of the NBS}

We used the NBS to identify sets of connections among wellestablished RSNs (Figure 1, Table 1) on which the depressed and nondepressed groups differed. The NBS identified one such set of connections: ( $p<0.024$, corrected; Figure 2). Specifically, this set of connections between RSNs was characterized by hypoconnectivity in the MDD group compared with the CTL group among the dorsal attention, cingulo-opercular salience, frontoparietal central executive, default mode, and somatomotor networks. The specific RSNs, quantification of their functional connectivity, and related group differences are presented in Table 2.

\section{Group Differences in Global and Local Graph Metrics}

There were no significant group differences in any global or local graph metrics following Bonferroni multiple comparisons correction (see Supplementary Tables S3 and S4).

\section{Results Using Anatomically Defined Nodes}

Using AAL atlas-based nodes, the NBS did not yield a group difference in connectivity $(p>0.10)$.

\section{Correlations Between Network Metrics and Measures of Depression and Age}

To assess relations between NBS-identified abnormal connectivity and clinical measures, for each MDD participant we averaged Fisher's transformed partial correlation coefficients across NBS-identified connections in which there was a significant group difference in functional connectivity and computed correlations with RADS-2 T-scores and depression duration. A significant negative correlation was obtained between depression duration and the average connectivity of the set of connections with reduced connectivity among attention, salience, default mode, and somatosensory RSNs (Table 2; Spearman's $\rho=-0.344$, $p=0.020$, corrected). Thus individuals who had longer duration of depression exhibited less connectivity among the RSNs that had reduced intranetwork connectivity in the MDD compared with the CTL group. To further explore this relation, we computed the correlations between depression duration and the connectivity between each pair of RSNs from the NBS-identified set of connections. These analyses yielded a significant negative correlation between depression duration and functional connectivity between dorsal attention networks (networks 5 and 6 in Figure 1 and Table 1; Spearman's $\rho=-0.425, p=0.003$, corrected). None of the other correlations was statistically significant, including correlations between network properties and age either across or within groups (see Supplementary Table S5 for results).

\section{Group Differences in Intranodal Connectivity}

Analysis of intranodal connectivity yielded a significant effect for one connection within the attention network (network 5), one of the networks showing reduced internetwork connectivity in our NBS analysis. Specifically, the MDD group exhibited reduced functional connectivity compared with the CTL group $(t(109)=3.05, p=0.003 ; M / S D$ : MDD $-0.050 / 0.163$; CTL $0.049 / 0.177)$. The regions implicated in this connectivity were bilateral components of the attention network located along the parieto-occipital sulcus at the 
juncture of dorsal occipital and ventral parietal cortex, spanning to temporal cortex (see Supplementary Figure S1 for surface renderings). No other connection for any other node exhibited a statistically significant group difference after Bonferroni correction.

\section{Replication of Connolly et al (2013) and Kaiser et al (2015)}

Additional seed-based analyses were conducted to assess replication of Connolly et al (2013) and Kaiser et al (2015). The results from Connolly et al (2013) were largely replicated in this overlapping and larger data set, including: (1) greater functional connectivity between sgACC and bilateral insulae in the MDD compared with the CTRL group; (2) greater functional connectivity between sgACC and right amgydala in the MDD compared with the CTL group; and (3) reduced connectivity between sgACC and precuneus in the MDD compared with the CTL group. For further details, see Supplementary Results 'Replication of Connolly et al (2013)' and Supplementary Table S6. The results of Kaiser et al (2015) replicated in the current data set include: (1) reduced intranetwork executive control network connectivity in the MDD compared with the CTL group; (2) increased intranetwork default mode network connectivity in the MDD compared with the CTL group; (3) decreased connectivity between executive control and dorsal attention networks in the MDD compared with the CTL group; (4) increased connectivity between executive control and default mode networks in the MDD compared with the CTL group; (5) decreased connectivity between the default mode and salience networks in the MDD compared with the CTL group; (6) decreased connectivity between the limbic and default mode networks in the MDD compared with the CTL group; and (7) decreased connectivity between the salience network and a seed in the precuneus (for details, see Supplementary Results 'Replication of Kaiser et al (2015)'; Supplementary Table S8).

\section{DISCUSSION}

This is the first study to use network-based analyses to assess abnormalities in connectivity among intrinsic RSNs in adolescents with MDD. Our investigation yielded several novel findings. First, relative to healthy controls, adolescents with MDD exhibited significantly reduced functional connectivity in a set of interconnected RSNs. Specifically, we found reduced functional connectivity among attention, central executive, salience, default mode, and somatosensory RSNs (Figure 2). MDD-related abnormalities in network connectivity were not identified when using anatomically defined nodes. Moreover, longer depressive duration was significantly associated with reduced connectivity among these RSNs and, in particular, with connections between dorsal attention networks. Previous studies have demonstrated that RSN connectivity matures during adolescent development, with neighboring connections weakening and distant connections strengthening over time, particularly in attention, central executive, salience, and default mode networks (Fair et al, 2007, 2008, 2009; Power et al, 2010). In this context, our results are consistent with the idea that adolescent MDD affects neurodevelopmental trajectories of functional networks in the form of hypoconnectivity between developing RSNs (Menon, 2013; Weir et al, 2012). Finally, we replicated results reported in prior resting-state work in both depressed adolescents and adults (Connolly et al, 2013; Kaiser $e t$ al, 2015), which suggests that a consistent pattern of abnormal connectivity manifests in MDD.

Contrary to our hypotheses, we did not observe group differences in network connections of limbic RSNs. Although this was surprising, it may be explained by findings that functional network development during adolescence is concentrated primarily in attention, central executive, salience, and default mode networks (Fair et al, 2009; Grayson et al, 2014; Kelly et al, 2009; Power et al, 2010). During early to mid-adolescence, the anterior cingulate cortex (ACC) and other anterior prefrontal cortical regions specifically reorganize and shift connectivity from frontoparietal central executive networks to cingulo-opercular salience networks (Fair et al, 2009; Power et al, 2010). Recent work has also demonstrated that, in attention, central executive, and default mode networks, shorter connections tend to be stronger in children and weaken over development, whereas longer-distance connections are relatively weak in children and strengthen with development (Fair et al, 2009; Kelly et al, 2009; Supekar et al, 2009). In contrast, limbic networks solidify early in development (Smyser et al, 2010; Yu et al, 2014). Thus our results offer initial evidence that adolescent MDD may affect the connectivity patterns of specific functional networks that are still undergoing development.

Our results are consistent with several findings from the adolescent MDD literature but are ultimately difficult to compare directly, given different methodological approaches and investigatory objectives. For example, while some of the resting-state studies in adolescent MDD provide evidence of reduced functional connectivity of nodes of several RSNs assessed in the present study, sample characteristics (age range, comorbidities, and medication status) may account for their report of opposing directionality of connectivity (Cullen et al, 2014; Davey et al, 2012). Of note, prior studies examining resting-state functional connectivity in adolescent MDD (Connolly et al, 2013; Cullen et al, 2014; Davey et al, 2012; Ho et al, 2015) did not examine connectivity between RSNs. Instead, these studies focused on assessing functional connectivity of a relatively small single seed region as a proxy for an entire network (e.g., dorsal ACC as a proxy for salience network) or used anatomically defined templates. Anatomically defined ROIs often span RSNs and thus may not have homogenous signal (Gordon et al, 2014). Moreover, depression onset, duration, and severity influence the development of functional networks (Meng et al, 2014; Tao et al, 2011; Zhang et al, 2011) and could contribute to the different presentations in adolescents compared with adults with MDD. Our approach of employing NBS and graph analyses to examine RSNs as functionally defined nodes and to investigate interactions among multiple RSNs is critical and relevant to the study of MDD in the adolescent brain, where network-level development is still ongoing (Fair et al, 2007; 2008; 2009; Power et al, 2010). From a neurodevelopmental perspective, our study of a sample of adolescents who are still undergoing development in functional networks may explain why we found overall reduced connectivity between RSNs in contrast to the findings of increased connectivity 
that are often reported in adult MDD (Greicius et al, 2007; Sheline et al, 2010; Tao et al, 2011; Zhang et al, 2011) and have also been reported in some seed-based functional connectivity studies of adolescent MDD (Connolly et al, 2013; Cullen et al, 2014; Davey et al, 2012; Ho et al, 2015; Pannekoek et al, 2014). Our findings also highlight the importance of considering neurodevelopmental factors when forming and testing neural models of MDD and potentially focusing on network-based outcomes as promising treatment targets.

Our results of reduced connectivity among several RSNs in the MDD vs CTL adolescents were obtained in the absence of significant group differences in global and local graph metrics. Thus our results do not match the preliminary graph theory results by Jin et al (2011), who reported reduced small-worldness and increased nodal degree in adolescents with MDD compared with controls. Our findings are consistent, however, with graph studies of adult depression in which small-worldness was preserved in patients with MDD (Bohr et al, 2012; Lord et al, 2012; Meng et al, 2014; Veer et al, 2010; Zhang et al, 2011). A recent diffusion-weighted imaging study of adult MDD used the NBS and assessed graph metrics of structural brain networks. This investigation also did not find significant group differences in global and local graph metrics, despite reporting significant group differences in the NBS-identified subnetworks (Korgaonkar et al, 2014). Given that NBS and graph metrics assess distinct aspects of network topology, discrepancies between NBS and global and local graph metric results are therefore not necessarily surprising.

The hypoconnectivity between RSNs that we observed in adolescents with MDD correlated significantly with longer depression duration but did not correlate with depression severity at the time of scan. Self-report measures of levels of depressive symptom severity, such as the RADS-2, assess mood state. Thus it may be that functional abnormalities between specific pairs of regions reflect depressive state, such as those results from seed-based studies that have been reported previously (Connolly et al, 2013; Cullen et al, 2014; Davey et al, 2012). Our finding of weaker connection strength among RSNs significantly correlating with greater depression duration may reflect a 'neural scar' or more long-term neurobiological marker of this disorder. Given that RSN connectivity maturation during adolescent development is in part characterized by strengthened connections among attention, central executive, salience, and default mode networks (Fair et al, 2009; Power et al, 2010), it is possible that our results reflect delayed neurodevelopment in adolescents with MDD. However, longitudinal studies are needed to test this possibility more explicitly and systematically.

Importantly, our results replicate previous resting-state functional connectivity work in MDD. Kaiser et al (2015) conducted a meta-analysis of functional connectivity in adolescent to elderly MDD across 25 publications that included 556 individuals with MDD and 518 healthy controls. It is noteworthy that we have replicated, using seed-based methods, the connectivity results reported (Kaiser et al, 2015). Additionally, we have also replicated Connolly et al (2013) in our overlapping and larger sample. The current replications indicate that the large-scale hypoconnectivity associated with the MDD compared with the CTL group that was quantified using the NBS co-occurs with network connectivity abnormalities that have been observed previously in individuals with MDD and have been identified using seed-based methods as applied by our group (Connolly et al, 2013) and using meta-analytic methods (Kaiser et al, 2015). Future research should further explicate the relations between large-scale hypoconnectivity and cognitive, affective, clinical, and behavioral abnormalities related to MDD. For example, our current results have implicated abnormalities of the attention, salience, and default mode networks in large-scale hypoconnectivity in adolescent MDD. Future research should assess whether this hypoconnectivity is related to specific aspects of cognition and affect associated with MDD and that may be related to the known functional roles of these networks.

By assessing functional connectivity intranodally, we have shown that adolescent MDD is associated with reduced connectivity within the dorsal visual attention network and, specifically, between bilateral regions spanning parietooccipital sulcus. This indicates that specific components of functional networks are concurrently abnormally connected in adolescent MDD. One possibility is that abnormal connectivity of regions near parieto-occipital sulcus in MDD is associated with inability to disengage from the visual dorsal stream. In a magnetoencephalography study of unselected adults by Tuladhar et al (2007), sources located in the vicinity of the parieto-occipital sulcus were associated with working memory load as tracked by activity in the alpha band (Tuladhar et al, 2007). The authors suggested that this is indicative of alpha activity underlying disengagement or inhibition of the visual dorsal stream. Indeed, alpha functional connectivity between prefrontal and sensory regions has previously been shown to be related to inhibition of irrelevant sensory stimuli (Sacchet et al, 2015a). In the context of MDD, this suggests that abnormal connectivity in these parieto-occipital regions may be related to robust biases in attention and inhibitory control of irrelevant sensory or internally generated information (for a review, see Gotlib and Joormann, 2010). Future research should assess this possibility more directly.

We also assessed group differences in network properties using the NBS and anatomically defined nodes using the AAL atlas (Tzourio-Mazoyer et al, 2002). This analysis did not yield group differences in network connectivity, in contrast to the results obtained using nodes derived from the functionally defined Yeo 17-network solution (Yeo et al, 2011). This inconsistency may be explained by the results of prior investigations that suggest that the AAL atlas has poorer signal homogeneity than do functionally defined ROIs (Craddock et al, 2011). The poor signal homogeneity of AAL nodes may be explained by the fact that anatomically defined nodes span multiple RSNs (Gordon et al, 2014). Thus the current results highlight that anatomically defined nodes may miss network abnormalities that occur within and between functionally defined networks.

Despite the strengths of the current investigation, including a large unmedicated sample and novel assessment of RSN interactions, the cross-sectional design of this study limits causal inferences. Longitudinal studies tracking at-risk individuals are essential to determine whether connectivity interactions among the identified RSNs indicate an increased risk for depression in healthy individuals. Similarly, longitudinal studies examining natural history or treatment 
effects may help determine whether these functional network abnormalities are reversible with successful treatment or, alternatively, represent a 'neural scar' of depression. The current study is also limited in the age range of participants (13-18 years) and is not adequately powered to assess the extent to which MDD alters the neurodevelopmental trajectory of functional RSNs. Future longitudinal studies will be essential to investigate these issues. Also, although we did not find differences in global or local graph metrics using univariate statistical methods, it is possible that machine learning methods may differentiate adolescents with MDD from healthy individuals (e.g., tractography-based network measures and adult MDD; Sacchet et al, 2014, 2015b). Furthermore, Bonferroni multiple comparison correction may be too conservative in the context of assessing betweengroup differences in graph metrics of functional networks (Meskaldji et al, 2013). An additional limitation is the use of regression to remove effects of the nuisance variable of WASI total scores (Miller and Chapman, 2001). Future research should assess larger samples that are matched on measures of intelligence. It is also noteworthy that Bonferroni correction may be too stringent for multiple comparison correction for the tests of global and local graph metrics and intranodal connectivity. Future, more targeted, assessment of these connectivity features is warranted. Finally, another explanation for the lack of group differences involving limbic networks is that the functional atlas used here includes only cortical regions (Yeo et al, 2011). Future research should assess whether including non-cortical regions in the RSNs identified here might influence large-scale interactions among RSNs.

In conclusion, using network-based approaches, we found reduced functional connectivity between attention, central executive, salience, and default mode RSNs in adolescents with MDD that have not previously been detected using seed-based resting-state fMRI methods. Because connectivity increases between these RSNs over normal development, our results suggest that MDD may adversely affect normal neurodevelopment during adolescence and that neural models of adolescent MDD should take neurodevelopmental factors into consideration. Network-based outcomes that go beyond activation of single brain areas may also offer more promising treatment targets. Finally, studies with longitudinal designs are needed to determine how RSN interactions relate to the etiology and progression of MDD during adolescence.

\section{FUNDING AND DISCLOSURE}

The authors declare no conflict of interest.

\section{ACKNOWLEDGMENTS}

This study was supported by NSF Integrative Graduate Education and Research Traineeship (IGERT) Recipient Award 0801700 to MDS; NSF Graduate Research Fellowship Program (GRFP) DGE-1147470 to MDS; NIMH Neuroscience Research Training Award T32 MH020016 to MDS; NIMH 7R01MH085734, 3R01MH085734-02S1 and R01MH08573405S1 to TTY; by the Brain and Behavior Research Foundation (formerly NARSAD) to TTY; by the American Foundation for
Suicide Prevention PDF-1-064-13 to TCH; by the Swedish Research Council 350-2012-303 to EHB; by the Center of Excellence in Stress and Mental Health; and by a Veteran's Affairs Merit Grant to ANS. We are grateful to Nisreen Mobayed, Jing Wu, Mary Luna, and Audrey Fortezzo for help with data collection and data management.

\section{REFERENCES}

Bai F, Shu N, Yuan Y, Shi Y, Yu H, Wu D et al (2012). Topologically convergent and divergent structural connectivity patterns between patients with remitted geriatric depression and amnestic mild cognitive impairment. J Neurosci 32: 4307-4318.

Bassett DS, Bullmore E (2006). Small-world brain networks. Neuroscientist 12: 512-523.

Birmaher B, Arbelaez C, Brent D (2002). Course and outcome of child and adolescent major depressive disorder. Child Adolesc Psychiatr Clin N Am 11: 619-637.

Birmaher B, Brent D (2007). Practice parameter for the assessment and treatment of children and adolescents with depressive disorders. J Am Acad Child Psychiatry 46: 1503-1526.

Bohr IJ, Kenny E, Blamire A, O'Brien JT, Thomas AJ, Richardson J et al (2012). Resting-state functional connectivity in late-life depression: higher global connectivity and more long distance connections. Front Psychiatry 3: 116.

Bullmore ET, Suckling J, Overmeyer S, Rabe-Hesketh S, Taylor E, Brammer MJ (1999). Global, voxel, and cluster tests, by theory and permutation, for a difference between two groups of structural MR images of the brain. IEEE Trans Med Imaging 18: 32-42.

Connolly CG, Wu J, Ho TC, Hoeft F, Wolkowitz O, Eisendrath S et al (2013). Resting-state functional connectivity of subgenual anterior cingulate cortex in depressed adolescents. Biol Psychiatry 74: 898-907.

Craddock RC, James GA, Holtzheimer PE III, Hu XP, Mayberg HS (2011). A whole brain fMRI atlas generated via spatially constrained spectral clustering. Hum Brain Mapp 33: 1914-1928.

Cullen KR, Westlund MK, Klimes-Dougan B, Mueller BA, Houri A, Eberly LE et al (2014). Abnormal amygdala resting-state functional connectivity in adolescent depression. JAMA Psychiatry 71: 1138-1147.

Davey CG, Harrison BJ, Yücel M, Allen NB (2012). Regionally specific alterations in functional connectivity of the anterior cingulate cortex in major depressive disorder. Psychol Med 42: 2071-2081.

Fair DA, Cohen AL, Dosenbach NUF, Church JA, Miezin FM, Barch DM et al (2008). The maturing architecture of the brain's default network. Proc Natl Acad Sci USA 105: 4028-4032.

Fair DA, Cohen AL, Power JD, Dosenbach NUF, Church JA, Miezin FM et al (2009). Functional brain networks develop from a "local to distributed" organization. PLoS Comput Biol 5: e1000381.

Fair DA, Dosenbach NUF, Church JA, Cohen AL, Brahmbhatt S, Miezin FM et al (2007). Development of distinct control networks through segregation and integration. Proc Natl Acad Sci USA 104: 13507-13512.

Fornito A, Zalesky A, Breakspear M (2013). Graph analysis of the human connectome: promise, progress, and pitfalls. Neuroimage 80: $426-444$.

Gong Q, He Y (2015). Depression, neuroimaging and connectomics: a selective overview. Biol Psychiatry 77: 223-235.

Gordon EM, Laumann TO, Adeyemo B, Huckins JF, Kelley WM, Petersen SE (2014). Generation and evaluation of a cortical area parcellation from resting-state correlations. Cereb Cortex 26: 288-303.

Gotlib IH, Joormann J (2010). Cognition and depression: current status and future directions. Annu Rev Clin Psychol 6: 285-312. 
Grayson DS, Ray S, Carpenter S, Iyer S, Dias TGC, Stevens C et al (2014). Structural and functional rich club organization of the brain in children and adults. PLoS One 9: e88297.

Greicius MD, Flores BH, Menon V, Glover GH, Solvason HB, Kenna $\mathrm{H}$ et al (2007). Resting-state functional connectivity in major depression: abnormally increased contributions from subgenual cingulate cortex and thalamus. Biol Psychiatry 62: 429-437.

Ho TC, Connolly CG, Blom EH, LeWinn KZ, Strigo IA, Paulus MP et al (2015). Emotion-dependent functional connectivity of the default mode network in adolescent depression. Biol Psychiatry 78: 635-646.

Jamison DT, Breman JG, Measham AR, Alleyne G, Claeson M, Evans DB et al (2006). Disease Control Priorities in Developing Countries. World Bank Publications: Washington, DC, USA.

Jin C, Gao C, Chen C, Ma S, Netra R, Wang Y et al (2011). A preliminary study of the dysregulation of the resting networks in first-episode medication-naive adolescent depression. Neurosci Lett 503: 105-109.

Kaiser RH, Andrews-Hanna A-HJ, Wager WT, Pizzagalli PD (2015). Large-scale network dysfunctionin major depressive disorder: a meta-analysis of resting-state functional connectivity. JAMA Psychiatry 72: 603-611.

Kelly AMC, Di Martino A, Uddin LQ, Shehzad Z, Gee DG, Reiss PT et al (2009). Development of anterior cingulate functional connectivity from late childhood to early adulthood. Cereb Cortex 19: $640-657$.

Kerestes R, Davey CG, Stephanou K, Whittle S, Harrison BJ (2010). Functional brain imaging studies of youth depression: a systematic review. Neuroimage Clin 4: 209-231.

Kessler RC, Walters EE (1998). Epidemiology of DSM-III-R major depression and minor depression among adolescents and young adults in the National Comorbidity Survey. Depress Anxiety 7: 3-14.

Korgaonkar MS, Fornito A, Williams LM, Grieve SM (2014). Abnormal structural networks characterize major depressive disorder: a connectome analysis. Biol Psychiatry 76: 567-574.

Lewinsohn PM, Rohde P, Seeley JR (1998). Major depressive disorder in older adolescents: prevalence, risk factors, and clinical implications. Clin Psychol Rev 18: 765-794.

Lord A, Horn D, Breakspear M, Walter M (2012). Changes in community structure of resting state functional connectivity in unipolar depression. PLoS One 7: e41282.

Meng C, Brandl F, Tahmasian M, Shao J, Manoliu A, Scherr M et al (2014). Aberrant topology of striatum's connectivity is associated with the number of episodes in depression. Brain 137: 598-609.

Menon V (2011). Large-scale brain networks and psychopathology: a unifying triple network model. Trends Cogn Sci 15: 483-506.

Menon V (2013). Developmental pathways to functional brain networks: emerging principles. Trends Cogn Sci 17: 627-640.

Meskaldji DE, Fischi-Gomez E, Griffa A, Hagmann P, Morgenthaler S, Thiran J-P (2013). Comparing connectomes across subjects and populations at different scales. Neuroimage 80: 416-425.

Miller GA, Chapman JP (2001). Misunderstanding analysis of covariance. J Abnorm Psychol 110: 40.

Mulders PC, van Eijndhoven PF, Schene AH, Beckmann CF, Tendolkar I (2015). Resting-state functional connectivity in major depressive disorder: a review. Neurosci Biobehav Rev 56: 330-344.

Pannekoek JN, van der Werff SJA, Meens PHF, van den Bulk BG, Jolles DD, Veer IM et al (2014). Aberrant resting-state functional connectivity in limbic and salience networks in treatment-naïve clinically depressed adolescents. J Child Psychol Psychiatry 55: $1317-1327$.
Power JD, Fair DA, Schlaggar BL, Petersen SE (2010). The development of human functional brain networks. Neuron 67: 735-748.

Reynolds WM, Mazza JJ (1998). Reliability and validity of the Reynolds Adolescent Depression Scale with young adolescents. J School Psychol 36: 295-312.

Rubinov M, Bullmore E (2013). Fledgling pathoconnectomics of psychiatric disorders. Trends Cogn Sci 17: 641-647.

Sacchet MD, LaPlante RA, Wan Q, Pritchett DL, Lee AKC, Hamalainen $\mathrm{M}$ et al (2015a). Attention drives synchronization of alpha and beta rhythms between right inferior frontal and primary sensory neocortex. J Neurosci 35: 2074-2082.

Sacchet MD, Prasad G, Foland-Ross LC, Thompson PM, Gotlib IH (2014). Elucidating brain connectivity networks in major depressive disorder using classification-based scoring. Proc IEEE Int Symp Biomed Imaging 11: 592-595.

Sacchet MD, Prasad G, Foland-Ross LC, Thompson PM, Gotlib IH (2015b). Support vector machine classification of major depressive disorder using diffusion-weighted neuroimaging and graph theory. Front Psychiatry 6: 21.

Sheline YI, Price JL, Yan Z, Mintun MA (2010). Resting-state functional MRI in depression unmasks increased connectivity between networks via the dorsal nexus. Proc Natl Acad Sci USA 107: 11020-11025.

Smyser CD, Inder TE, Shimony JS, Hill JE, Degnan AJ, Snyder AZ et al (2010). Longitudinal analysis of neural network development in preterm infants. Cereb Cortex 20: 2852-2862.

Supekar K, Musen M, Menon V (2009). Development of large-scale functional brain networks in children. PLos Biol 7: e1000157.

Tao H, Guo S, Ge T, Kendrick KM, Xue Z, Liu Z et al (2011). Depression uncouples brain hate circuit. Mol Psychiatry 18: 101-111.

Tuladhar AM, Huurne NT, Schoffelen J-M, Maris E, Oostenveld R, Jensen O (2007). Parieto-occipital sources account for the increase in alpha activity with working memory load. Hum Brain Mapp 28: 785-792.

Tzourio-Mazoyer N, Landeau B, Papathanassiou D, Crivello F, Etard O, Delcroix N et al (2002). Automated anatomical labeling of activations in SPM using a macroscopic anatomical parcellation of the MNI MRI single-subject brain. Neuroimage 15: 273-289.

Veer IM, Beckman CF, van Tol M-J, Ferrarini L, Milles J, Veltman DJ et al (2010). Whole brain resting-state analysis reveals decreased functional connectivity in major depression. Front Syst Neurosci 4: 41.

Weir JM, Zakama A, Rao U (2012). Developmental Risk I: depression and the developing brain. Child Adolesc Psychiatric Clin N Am 21: 237-259.

Ye M, Yang T, Qing P, Lei X, Qiu J, Liu G (2015). Changes of functional brain networks in major depressive disorder: a graph theoretical analysis of resting-state fMRI. PLoS One 10: e0133775.

Yeo BT, Krienen FM, Sepulcre J, Sabuncu MR, Lashkari D, Hollinshead $M$ et al (2011). The organization of the human cerebral cortex estimated by intrinsic functional connectivity. J Neurophysiol 106: 1125-1165.

Yu Q, Peng Y, Mishra V, Ouyang A, Li H, Zhang H et al (2014). Microstructure, length, and connection of limbic tracts in normal human brain development. Front Aging Neurosci 6: 288.

Zalesky A, Fornito A, Bullmore ET (2010). Network-based statistic: Identifying differences in brain networks. Neuroimage 53: 1197-1207.

Zhang J, Wang J, Wu Q, Kuang W, Huang X, He Y et al (2011). Disrupted brain connectivity networks in drug-naive, firstepisode major depressive disorder. Biol Psychiatry 70: 334-342.

Supplementary Information accompanies the paper on the Neuropsychopharmacology website (http://www.nature.com/npp) 